\title{
Admissibility Intervals for Linear Correlation Coefficients
}

\section{Luisa Canal, Rocco Micciolo}

Department of Cognitive Sciences and Education

University of Trento

Via Matteo del Ben, 5

38068 - Rovereto (TN) - Italy

(e-mail: luisa.canal@unitn.it)

The correlation coefficient is widely used to quantify the degree of association between two quantitative variables. By resorting to the geometric representation of the linear correlation coefficient, it is possible to calculate the upper and lower bounds of the correlation coefficient between two variables $x_{1}, x_{2}$ when the correlation coefficients with a third variable $x_{3}$ are available. Implications in observational studies, where $x_{3}$ could be a proxy of a target variable $x_{2}$, whose direct measurement is too expensive or impractical, are discussed.

Key words Geometric representation, observational study, proxy variable 


\section{Introduction}

In observational studies, particularly in the epidemiological field, correlations are often used to summarize the results by expressing the relationships between two continuous variables, say $x_{1}$ and $x_{2}$. For example, $x_{1}$ may be a biological variable related to the risk of developing a disease (such as cholesterol or triglyceride plasma levels or blood pressure) and $x_{2}$ a variable possibly associated with $x_{1}$ (for example, total body fat, total energy intake, personal exposure to a pollutant). However, a direct measurement of $x_{2}$ may be too expensive or impractical (for example total body fat could be evaluated using dual energy X-ray absorptiometry, underwater weighing or total body potassium; total energy intake with indirect calorimetry or daily recorded dietary intake). In this case the researcher usually resorts to another variable $x_{3}$, that can be considered a proxy of the target variable $x_{2}$ (for example, body mass index or skinfold thickness measured at various body locations; food frequency questionnaires; air quality measures obtained from centrally located outdoor monitoring stations). As a result, the analysis is based on the correlation coefficient $r_{13}$ between $x_{1}$ and the proxy $x_{3}$, instead of the correlation coefficient $r_{12}$ between $x_{1}$ and the target $x_{2}$. Obviously, in general, $r_{13} \neq r_{12}$.

It is well known that, for fixed values of $r_{13}$ and $r_{23}, r_{12}$ cannot take any value between -1 and +1 , but does have upper and lower bounds depending on $r_{13}, r_{23}$. In fact, the correlation matrix $\mathbf{R}$ between $x_{1}, x_{2}, x_{3}$ must be semi-definite positive (i.e. its eigenvalues must be all non negatives). Therefore, for fixed values of $r_{13}$ and $r_{23}$, there exist only a limited interval of values admissible for $r_{12}$; it is possible to find this interval from the solutions of the characteristic equation associated with $\mathbf{R}$, seeking for what values of $r_{12}$ all the solutions (i.e. the eigenvalues) are non-negative. Even if the solutions of the characteristic equation (which is of third grade) must be all real (since $\mathbf{R}$ is symmetric), they are somewhat cumbersome to calculate in the general case. 
On the contrary, using a geometric approach it is possible to give a simple, general, answer to such a question.

\section{A geometric solution}

It is well known that the correlation coefficient can be geometrically interpreted as the cosine of an angle; more precisely, the correlation coefficient $r_{x y}$ between the variables $x$ and $y$ is the cosine of the angle $\alpha_{x y}$ between their representative vectors $\mathbf{v}_{x}$ and $\mathbf{v}_{y}: r_{x y}=\cos \left(\alpha_{x y}\right)$. This relationship can be reversed; if one knows the value of $r_{x y}$, it is possible to found the corresponding angle: $\alpha_{x y}=\cos ^{-1}\left(r_{x y}\right)$.

Let $\mathbf{v}_{1}, \mathbf{v}_{2}, \mathbf{v}_{3}$ denote three vectors (which represent the variables $\left.x_{1}, x_{2}, x_{3}\right)$ separated by the angles $\alpha_{12}, \alpha_{13}, \alpha_{23}$. Let $\mathbf{v}_{1}$ and $\mathbf{v}_{3}$ be linearly independent (i.e. $\left|r_{13}\right| \neq 1$ ) and let $\mathbf{v}_{2}$ be linearly dependent from $\mathbf{v}_{1}, \mathbf{v}_{3}$. In this case, as shown by Leung and Lam (1975), the angle $\alpha_{12}$ between $\mathbf{v}_{1}$ and $\mathbf{v}_{2}$ can take only two values: $\alpha_{13}-\alpha_{23}$ or $\alpha_{13}+\alpha_{23}$ when $\alpha_{13}+\alpha_{23} \leq 180^{\circ}$ and $\alpha_{13}-\alpha_{23}$ or $360^{\circ}-\left(\alpha_{13}+\alpha_{23}\right)$ when $\alpha_{13}+\alpha_{23} \geq 180^{\circ}$. If $\mathbf{v}_{2}$ is not linearly dependent from $\mathbf{v}_{1}, \mathbf{v}_{3}$, the angle $\alpha_{12}$ can take all the values between the two defined above. In either case:

$$
\cos \left(\alpha_{13}+\alpha_{23}\right) \leq r_{12} \leq \cos \left(\alpha_{13}-\alpha_{23}\right)
$$

From this inequality it is possible to calculate the interval of admissible values for the correlation coefficient $r_{12}$; we call this interval the admissibility interval for $r_{12}$.

The inequality in equation (1) describes an elliptical region in the $\left(r_{13}, r_{12}\right)$-plane if $r_{23}$ is held fixed. In fact, it is possible to rewrite equation (1) as

$$
r_{13} r_{23}-\sqrt{\left(1-r_{13}^{2}\right)\left(1-r_{23}^{2}\right)} \leq r_{12} \leq r_{13} r_{23}+\sqrt{\left(1-r_{13}^{2}\right)\left(1-r_{23}^{2}\right)}
$$

The boundary of this region is given by $r_{12}^{2}-2 r_{23} r_{12} r_{13}+r_{13}^{2}=1-r_{23}^{2}$. The length of the semi-major axis is $\sqrt{1+\left|r_{23}\right|}$ and points in the direction $\left(\operatorname{sgn}\left(r_{23}\right),+1\right)$, while the length 
of the semi-minor axis is $\sqrt{1-\left|r_{23}\right|}$ and points in the direction $\left(\operatorname{sgn}\left(r_{23}\right),-1\right)$.

\section{An illustration}

Let us consider the case $r_{23}=.95$ (i.e. an high positive correlation between the target variable $x_{2}$ and its proxy $x_{3}$ ) and $r_{13}=.70$. Inequality (2) becomes

$$
0.665-\sqrt{0.51 \times 0.0975} \leq r_{12} \leq 0.665+\sqrt{0.51 \times 0.0975}
$$

and the admissibility interval for the linear correlation coefficient $r_{12}$ between the variables $x_{1}$ and $x_{2}$ is $(.442, .888)$ in the case considered.

This example shows that, even in presence of an "high" correlation coefficient $r_{23}$ between the target variable $x_{2}$ and its proxy $x_{3}$, the true correlation $r_{12}$ between $x_{1}$ and $x_{2}$ could be considerably lower than the value $r_{13}$ observed between $x_{1}$ and $x_{3}$.

\section{Ellipses of admissibility}

Using inequality (2), it is possible to calculate, for fixed values of $r_{23}$, the admissibility interval for $r_{12}$ with respect to $r_{13}$ and to plot them. Figure 1 shows the results obtained for four selected values of $r_{23}(.8, .9, .95, .99)$. For a given value of $r_{13}$ on the abscissa, it is possible to read on the ordinate the upper and the lower bounds within which $r_{12}$ must be contained to satisfy the positive-definite constraint.

As the value of $\left|r_{23}\right|$ increases, the length of the minor axis of the ellipse decreases; as $\left|r_{23}\right|$ approaches one, the ellipse degenerates into the line segment from $[-1,-1]$ to $[1,1]$.

Inequality (2) implies a note of caution in interpreting the correlation $r_{13}$. In fact, as a consequence of this inequality, it is possible that, while the correlation $r_{13}$ found between $x_{1}$ and $x_{3}$ may be, for example, positive, the correlation coefficient $r_{12}$ between $x_{1}$ and $x_{2}$ may be zero (or even negative). 


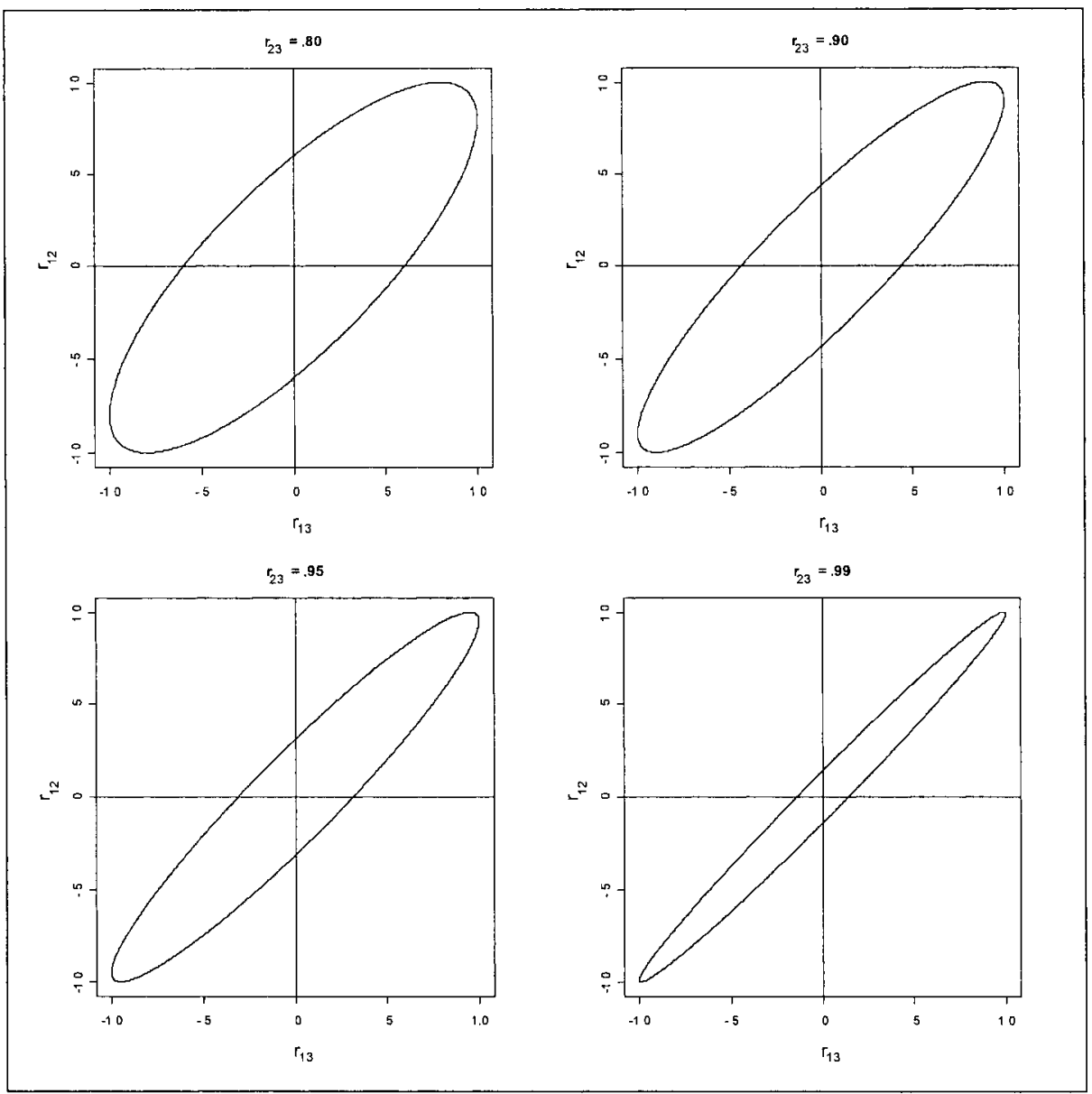

Figure 1. Admissible values for $r_{12}$ with respect to $r_{13}$ for selected values of $r_{23}$.

This is graphically exemplified in figure 2, where the shaded area shows the range of $r_{13}$ values for which the admissibility interval for $r_{12}$ includes zero (when $r_{23}=.9$ ); in this case this happens in the range $(-.436,+.436)$ of $r_{13}$.

For a given value of the correlation coefficient $r_{23}$ between $x_{2}$ and its proxy $x_{3}$, it is possible to deduce algebraically this range from (2). Let $k=\sqrt{1-r_{23}^{2}}$; then if $-k \leq r_{13} \leq+k$, the admissibility interval (1) for $r_{12}$ includes zero. 


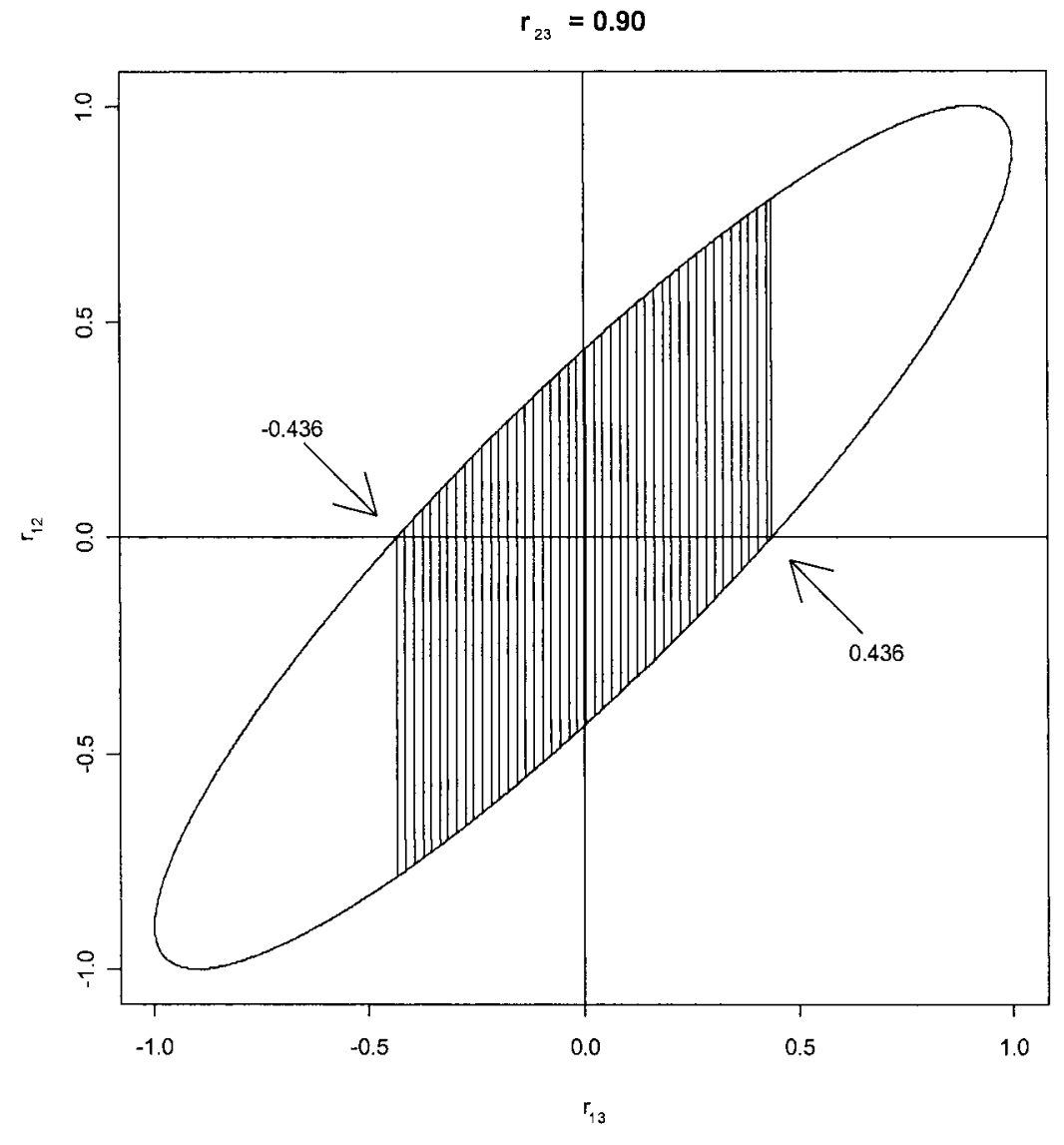

Figure 2. Admissible values for $r_{12}$ with respect to $r_{13}$ when $r_{23}=.9$. The dashed region shows $r_{13}$ values for which the admissibility interval for $r_{12}$ includes zero.

If $r_{23}=.95$ this occurs when $r_{13}$ is between -.312 and +.312 , while if $r_{23}=.99$ this occurs when $r_{13}$ is between -.141 and +.141 . If the correlation $r_{23}$ is .8 , observed values of $r_{13}$ between -.6 and +.6 are consistent with a correlation coefficient $r_{12}$ equal to zero or even with an opposite sign with respect to that found for $r_{13}$. The fact that $r_{12}$ and $r_{13}$ may have opposite signs is reminiscent of situation where the line of regression may be opposite to that of the correlation coefficient when there are correlated errors in both variables (McCartin, 2005). 


\section{Concluding remarks}

Although the results presented here are based on fundamental relationships who have been known for many years, their practical implications, particularly in the epidemiological field, are not routinely included in the interpretation of the results. It is possible that both the variability and the difficulties in reproducing the results of epidemiological studies could be, at least in part, due to the difference between the observed correlation with a variable which is a proxy of another target variable and the true correlation with such a target variable.

These results give a quantitative meaning to the prescription of using a proxy highly correlated with the target variable. On the other hand, they indicate that some caution is needed in interpreting low but significant correlations found in empirical studies.

The results presented here are intended to be descriptive and do not give a direct answer to the evaluation of the correlation with the target variable when only a proxy is available. However, by resorting to a geometric representation, an indirect answer is possible. The formulas presented are easily applicable in practice and can be helpful in the critical analysis and in the comparison of the results of empirical studies.

\section{Acknowledgment}

The authors are grateful to an anonymous referee for his useful suggestions.

\section{References}

Leung, C.K., Lam, K. (1975) A note on the geometric representation of the correlation coefficients, The American Statistician, 29, 128-130.

McCartin, B.J. (2005) The geometry of linear regression with correlated errors, Statistics, 39, 1-11. 